\title{
Thoracic Aortic Pseudoaneurysm Due to Screw Dislodgment Following Anterior Spinal Approach. Complex Surgical Management of Both Issues: A Case Report and Review of Literature
}

\author{
ROSA M. EGEA-GÁMEZ, MD, PHD, ${ }^{1}$ DAVID FERREÑO-MÁRQUEZ, MD, ${ }^{2}$ \\ LUIS DE BENITO FERNÁNDEZ, MD, PHD ${ }^{3}{ }_{\text {JOSE LOSADA-VIÑAS, MD, PHD, }}{ }^{2}$ \\ RAFAEL GONZÁLEZ-DÍAZ, MD, PHD ${ }^{1}$ \\ ${ }^{I}$ Department of Orthopaedic Surgery, Spinal Unit, Hospital Universitario Niño Jesús, Madrid, Spain, ${ }^{2}$ Department of Orthopaedic Surgery, Hospital Fundación \\ Alcorcón, Madrid, Spain, ${ }^{3}$ Department of Vascular Surgery, Hospital Fundación Alcorcón, Madrid, Spain
}

\begin{abstract}
A few descriptions about anterior thoracic arthrodesis causing thoracic aortic pseudoaneurysms due to late screw loosening are mentioned in the literature. We report a case that describes a hybrid approach complicated with an aortic injury when removing a screw from the aortic wall. A 57-year-old man was initially operated on for scoliosis due to poliomyelitis using dorsal thoracolumbar in situ fusion at an early age. At adulthood, the patient complained of spinal cord compression and severe myelopathy due to D9-D10 nonunion, and the patient required a double surgical approach. Almost a year later, he was diagnosed with a thoracic aneurysm caused by late screw loosening from the anterior plate. A hybrid approach was used to treat the aortic pseudoaneurysm (endograft stent) and for anterior vertebral hardware removal (rethoracotomy). Although an aortic stent was covering the aortic lumen, during the open part of the procedure, an aortic injury took place when removing the screw, requiring quick cross clamping and repair. Anterior vertebral hardware removal after a previous anterior spinal approach is a technically highly demanding procedure. As unexpected life-threatening complications can occur, this procedure should be performed in a setting with the capacity for both endovascular and open aortic repair.
\end{abstract}

Complications

Keywords: Thoracic aortic pseudoaneurysm, screw dislodgment, anterior spinal approach, aortic injury, spinal fusion

\section{INTRODUCTION}

Spinal stabilization surgery following scoliosis in adults is a complex surgery due to the characteristics of the patients, who frequently have multiple diseases, and the pathology of the spinal column itself. $^{1}$ The primary procedure for correcting large deformities of the spinal column consists of a posterior approach using an extensive arthrodesis with transpedicular screws and rods extending from lumbosacral to thoracic or even cervical levels. In some cases, a double approach is required to increase the stability of the system. Among the complications of this type of surgery are infection of the fixation devices, infection or dehiscence of the surgical wound, presence of pseudoarthrosis, device migration, and nervous or vascular complications. Formation of a pseudoaneurysm and rupture of the aorta are rare and serious compli- cations of difficult management. There are few reported cases in the literature, ${ }^{2}$ most involving pseudoaneurysms and/or aortic ruptures at the lumbar level treated with endograft stents, patches, or direct suture.

We here present a new case of pseudoaneurysm and rupture of the descending thoracic aorta by iatrogenic perforation of an anterior arthrodesis plate fixation screw, which required a hybrid approach: an endograft stent to repair the aortic pseudoaneurysm and rethoracotomy for screw removal.

\section{CASE PRESENTATION}

The patient was a 57-year-old male with a history of treated atrial fibrillation and ventricular tachycardia and poliomyelitis during childhood resulting in severe right thoracic kyphoscoliosis, which was 
operated on in 2014 via a posterior T8-L2 arthrodesis with transpedicular screws and rods.

The patient came to our center after falling down the stairs 2 months earlier and since then suffering progressive loss of strength in lower limbs and hypoesthesia in dermatomes below T10.

The magnetic resonance imaging scan revealed spinal canal stenosis with spinal cord compression at the level of T10-T11 associated with fracture of the fusion mass.

\section{First Surgery and Postoperative Course}

Based on clinical and radiological findings, we performed removal of the osteosynthesis material at that level, debridement of the fracture focus, posterior T10-T11 spinal cord decompression via the dorsal approach, fixation of transpedicular screws, and filling with bone allograft to improve the consolidation process. No complications occurred during the postoperative period, with the patient experiencing a marked improvement in sensory and motor symptoms in the lower limbs. On follow-up at 6 months and 1 year, computed tomography (CT) findings revealed the presence of pseudoarthrosis in the previously operated area (T10-T11), without breakage of the material, associated with symptoms of persistent lumbar pain, so it was decided to opt for a new surgical stabilization.

\section{Second Surgery and Postoperative Course}

An anterior thoracic spinal approach via extensive left posterolateral thoracotomy at the level of the fifth intercostal space was used to perform debridement and curettage of the area of pseudoarthrosis from T10 to T11, filling with mesh and bone allograft, and placement of an anterior arthrodesis plate with 2 proximal screws in T10 and 2 distal screws in T11 secured to the vertebral bodies. The patient experienced no complications during the immediate postoperative period, and the follow-up CT scan at 1 year revealed total vertebral fusion, with absence of any symptoms. However, at 11 months from the last surgery, the patient developed hemoptysis lasting for 2 weeks, without chest pain, fever, or sensation of dysthermia. The patient reported a sensation of retrosternal oppression and burning that worsened after intake, with a weight loss of $6 \mathrm{~kg}$, hyporexia, and asthenia. The plain chest $\mathrm{x}$-ray revealed enlargement of soft tissues in the left paravertebral region proximal to the aorta wall (Figure 1a, b). After excluding hemoptysis foci and suspected bleeding of vascular origin, it was decided to perform a CT angiography. This revealed a $3.4 \times 8.3 \times 3.1-\mathrm{cm}$ pseudoaneurysm of the descending thoracic aorta related to dislodgement of the lower left screw of $\mathrm{T} 11$ from the anterior arthrodesis plate (Figure 2a, b).

\section{Third Surgery and Postoperative Course}

The vascular surgery team treated the iatrogenic pseudoaneurysm endovascularly by placing a $28 \times 100$-mm endograft, normally positioned and without leakage in the follow-up CT (Figure 3), with its distal end in close contact with the screw of the anterior arthrodesis plate, resulting in complete disappearance of hemoptysis symptoms. No postoperative complications occurred. In a joint session with the vascular surgery team and the Spinal Unit of the Department of Orthopedic Surgery and Traumatology of our center, it was decided to remove the anterior arthrodesis plate and screws since (1) there was complete anterior and posterior vertebral fusion at the T10-T11 level and (2) a high risk remained of dislodgment of the lower left screw of T11, with formation of a new pseudoaneurysm or rupture despite the endograft stent.

\section{Fourth Surgery and Postoperative Course}

On a scheduled basis, the surgical team of our hospital (general surgery, vascular surgery, and traumatology spinal units) performed an extensive left rethoracotomy over the scar of the previous surgery. Following careful release of pulmonary adhesions and the aortic bed, the dislodged screw was visualized intraoperatively to be in contact with the endograft stent in its most distal portion. On removal of the osteosynthesis material, profuse bleeding occurred at the site of perforation of the screw, so clamping of the aorta proximal and distal to the site of bleeding and sectioning of the aorta through the endograft stent were performed. Screw removal was performed, but the rest of the material could not be removed due to osteointegration. The aorta was reconstructed by direct end-to-end suture of the endograft stent (Figure 4). There were no significant findings during the postoperative period until day 13 , on which the patient began to experience progressive anemization and dyspnea. An emergency simple x-ray and $\mathrm{CT}$ of the chest were performed, showing massive 

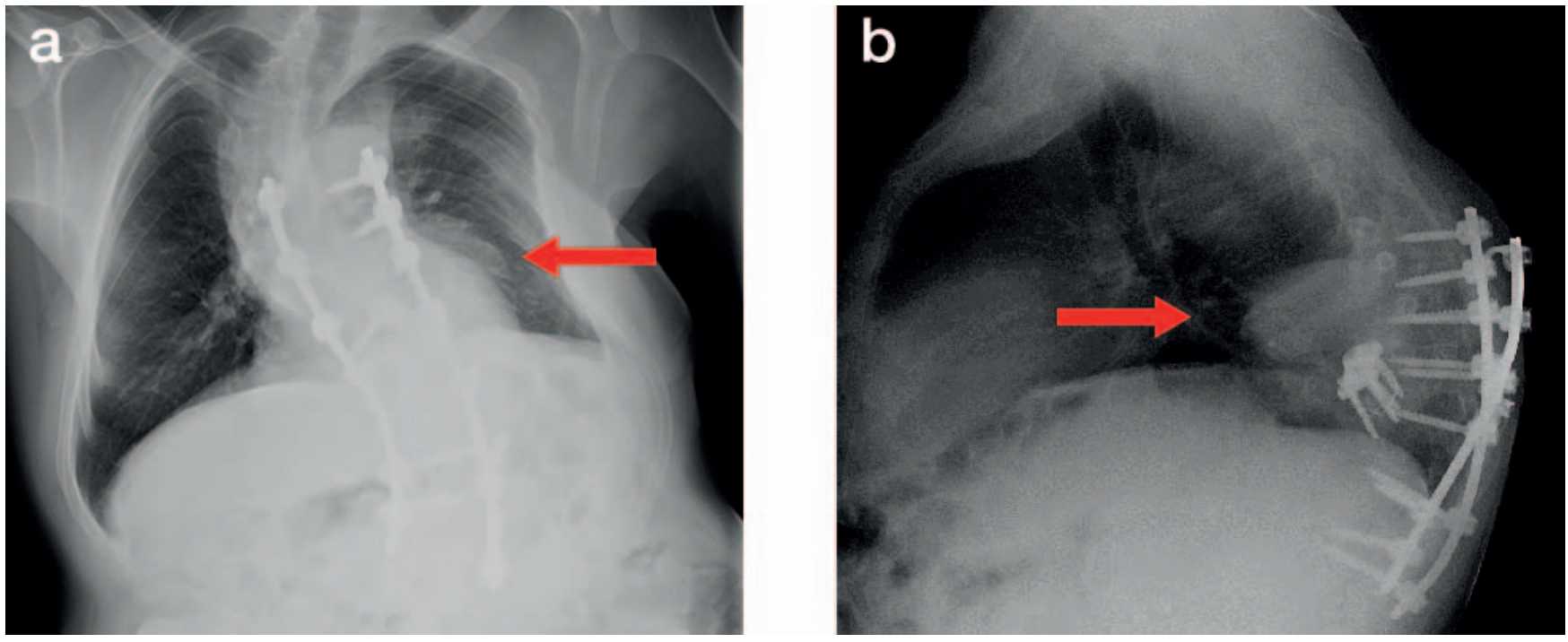

Figure 1. Plain chest X-ray revealed enlargement of soft tissues in the left paravertebral region proximal to the aorta wall.

left hemothorax $(90 \%$ of left pleural surface) (Figure 5), and it was opted to perform emergency surgery.

\section{Fifth Surgery and Postoperative Course}

Via extensive left rethoracotomy over the previous scar, the Department of General Surgery performed evacuation of approximately $2 \mathrm{~L}$ of blood clots, visualizing intraoperatively active bleeding through a branch of the intercostal artery. Hemostasis of the bleeding vessel was performed. No complication arose during the subsequent period, and the patient was discharged 14 days after reoperation for bleeding.

\section{DISCUSSION}

The number of anterior thoracic spinal procedures used in the treatment of a great variety of conditions involving the spinal column has increased in the past 20 years. ${ }^{3,4}$ At the same time, thoracic aortic injury after spinal instrumentation is still rarely documented in the literature. ${ }^{5}$ Nevertheless, there is a risk of injury after anterior spinal instrumentation due to the anterolateral position of the aorta with respect to the vertebral bodies; screws may lie $<2 \mathrm{~mm}$ from the aorta. ${ }^{6}$ It has been reported that most of these cases are recognized $<18$ months the spinal surgery is completed. Several methods of aortic repair have been pro-
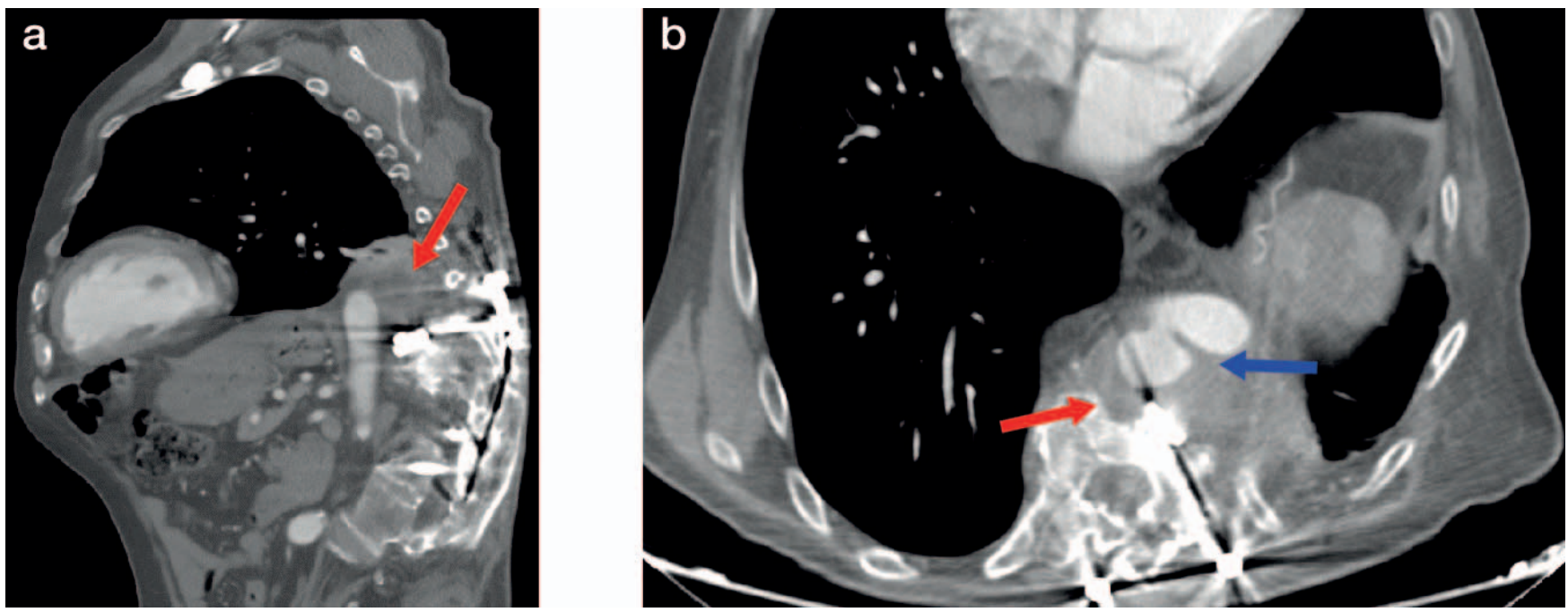

Figure 2. Pseudoaneurysm of the descending thoracic aorta related to dislodgement of the lower left screw of T11 from the anterior arthrodesis plate. 


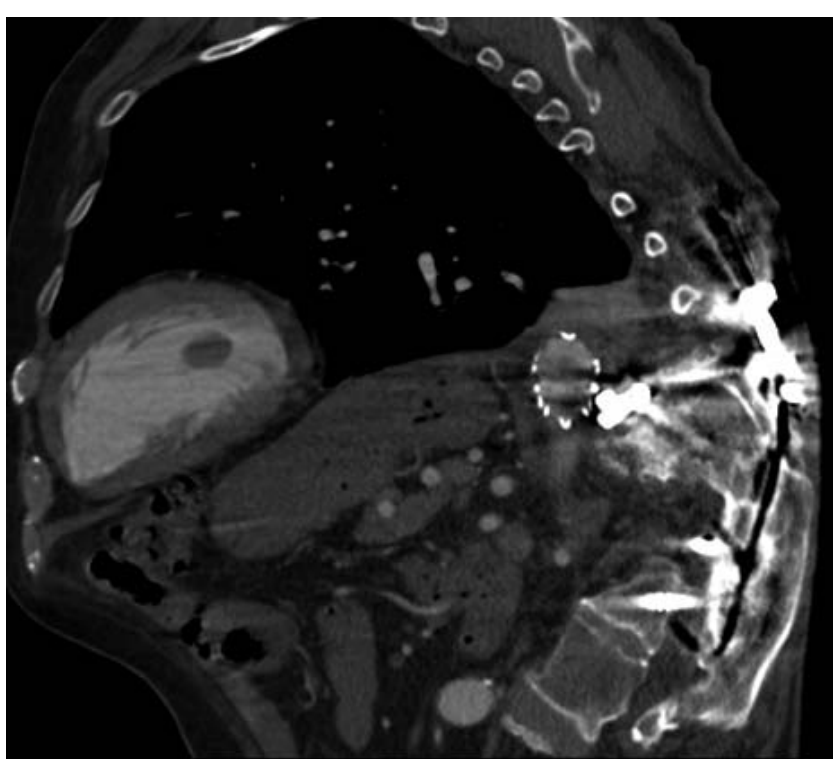

Figure 3. Pseudoaneurysm treated endovascularly by placing a $28 \mathrm{~mm} \times 100$ $\mathrm{mm}$ endograft, normally positioned and without leakage in the follow-up CT.

posed, including endograft stent grafting, patch angioplasty, and prosthetic graft replacement..$^{8-11}$ Hybrid approaches (endovascular followed by an open thoracic procedure) have also been described. ${ }^{12,13}$

To our knowledge, there are no cases reported in the literature documenting a hybrid approach complicated by an aortic injury when removing a screw from the aortic wall. This issue was successfully managed by performing an aortic end-to-end open repair, cross clamping the thoracic aorta below and above the perforation, and preserving the stent. At the time that the aorta was cross clamped, the screw was also removed. Neither cardiopulmonary bypass nor prosthetic graft was used.

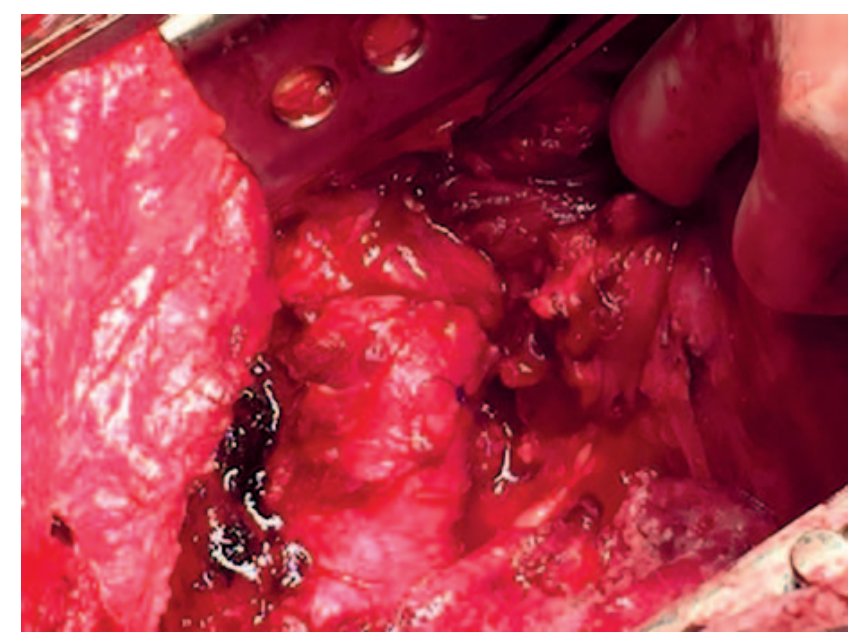

Figure 4. Aorta reconstruction by direct end-to-end suture of the endograft stent.

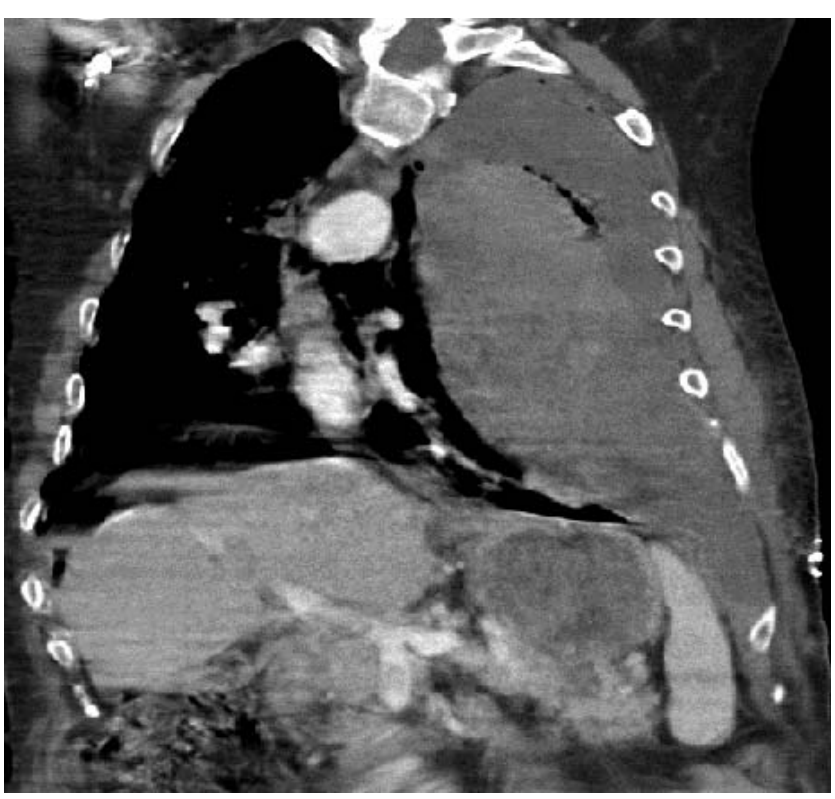

Figure 5. CT of the chest showing massive left hemothorax $(90 \%$ of left pleural surface).

Bavare et $\mathrm{al}^{12}$ described a similar case in which hemoptysis was also the initial symptom but in the immediate postoperative period. In this case, due also to an acute left hemothorax and hemodynamic instability, the hybrid procedure was performed on an emergency basis. The aorta was primarily repaired, but the screw was not removed. Their patient required a prosthetic replacement 5 months later due to an aortic rupture, and the screw was then removed.

The patient described in our case presented almost 18 months after his anterior spinal hardware was implanted. We first needed to control his hemoptysis and repair his pseudoaneurysm, which is why we initially managed the case with an aortic endograft in order to stabilize both issues. We considered screw removal the next step and final treatment to prevent pseudoaneurysm long-term growth ${ }^{14}$ or even aortic rupture.

We thought that it would be safe to remove the screw and that the aortic wall, with the stent covering its lumen, would prevent it from perforating during its removal, as shown in a similar case described by Colvard et al. ${ }^{13}$ The screw in our case was very close to the stent's lower edge, and when trying to remove it from the aorta wall, this unexpectedly ruptured. It was of great importance that (1) the aorta had been previously completely dissected far above and below the screw so that cross clamping was feasible, and that (2) the patient was fit enough to tolerate cross clamping. We would 
like to highlight the technical difficulties found to reach the aortic operative field due to severe lung adhesions to the chest wall and diaphragm. We also had to manage very dense fibrosis and scar tissue connecting the aorta to the anterior vertebral bodies. Both technical difficulties were caused by the patient's previous anterior spinal surgery through a left thoracotomy in which the lung and the aorta had to be manipulated and mobilized for placement of the vertebral hardware. We would also like to highlight the fact that an emergency thoracic aortic repair could be done end to end after cross clamping the aorta, cutting it at the level of the perforation, mobilizing it, removing the screw, and suturing back both ends through the stent wall. In this way, the time for cross clamping was reduced, and there was no need for heparin, use of a prosthetic graft, or left atrial femoral bypass.

A more posterior instrumentation position in the vertebral body decreases the risk of vascular complications of the great vessels, as has been shown in different studies that analyzed the position of the great vessels with respect to the vertebral body. ${ }^{15}$

\section{CONCLUSIONS}

Endovascular stent management of aortic pseudoaneurysms secondary to osteosynthesis material is not sufficient in most cases, so it is necessary to remove the anterior instrumentation. Anterior vertebral hardware removal after a previous anterior spinal approach is a technically highly demanding procedure. Unexpected life-threatening complications can occur when removing screws that are damaging the aortic wall, even when endograft stents have been placed to prevent this from happening. Spinal hardware removal should be performed in a setting with the capacity for both endovascular and open aortic repair. A more posterior instrumentation position in the vertebral body decreases the risk of vascular complications of the great vessels.

\section{REFERENCES}

1. Zanirato A, Damilano M, Formica M, et al. Complications in adult spine deformity surgery: a systematic review of the recent literature with reporting of aggregated incidences. Eur Spine J. 2018;27:2272-2284. doi:10.1007/s00586-018-5535-y

2. Pillai ST, Schoenhagen P, Subrahmanyan L, et al. Aortic dissection associated with penetration of a spinal pedicle screw: a case report and review of the literature. J Card Surg. 2014;29(3):377-381. doi:10.1111/jocs. 12327

3. Johnson JP, Pare LS, Torres RA. Thoracolumbar body replacement: material and techniques. Contemp Neurosurg. 1989;20(6):1-9.

4. Sundaresan N, Steinberger AA, Moore F, et al. Indications and results of combined anterior-posterior approaches for spine tumor surgery. J Neurosurg. 1996;85(3):438446. doi:10.3171/jns.1996.85.3.0438

5. Oskouian RJ Jr, Johnson JP. Vascular complications in anterior thoracolumbar spinal reconstruction. J Neurosurg. 2002;96(suppl 1):1-5. doi:10.3171/jns.2002.96.1.0001

6. Been HD, Kerkhoffs GM, Balm R. Endovascular graft for late iatrogenic vascular complication after anterior spinal instrumentation: a case report. Spine. 2006;31(22):E856-858. doi:10.1097/01.brs.0000240761.13090.a9

7. Kokotsakis J, Misthos P, Sakellaridis T, et al. Emergent endovascular repair as damage control step of aortic injury after posterior spinal instrumentation. Ann Thorac Surg. 2010;89(4):1304. doi:10.1016/j.athoracsur.2009.07.087

8. Carmignani A, Lentini S, Acri E, et al. Combined thoracic endovascular aortic repair and neurosurgical intervention for injury due to posterior spine surgery. J Card Surg. 2013;28(2):163-167. doi:10.1111/jocs.12052

9. Kakkos SK, Shepard AD. Delayed presentation of aortic injury by pedicle screws: report of two cases and review of the literature. J Vasc Surg. 2008;47(5):1074-1082. doi:10.1016/j.jvs. 2007.11.005

10. Sandhu HK, Charlton-Ouw KM, Azizzadeh A, Estrera AL, Safi HJ. Spinal screw penetration of the aorta. J Vasc Surg. 2013;57(6):1668-1670. doi:10.1016/j.jvs.2012.10.087

11. Xenos ES, Abedi NN, Davenport DL, et al. Metaanalysis of endovascular vs open repair for traumatic descending thoracic aortic rupture. J Vasc Surg. 2008;48(5):1343-1351. doi:10.1016/j.jvs.2008.04.060

12. Bavare C, Kim M, Blackmon S, Ellsworth W, Davies MG, Reardon MJ. Delayed aortic rupture after aortic endograft placement in patient with spinal hardware. Ann Thorac Surg. 2001;92(4):1512-1514. doi:10.1016/j.athoracsur. 2011.04.041

13. Colvard BD, Anaya-Ayala JE, Smolock CJ, Lumsden AB, Reardon MJ, Davies MG. Hybrid approach for removal of an errant intra-vascular pedicle spinal fixation screw in the thoracic aorta. J Vasc Surg. 2012;56(1):201-204. doi:10.1016/j. jvs.2011.12.084

14. Minor ME, Morrissey NJ, Peress R, et al. Endovascular treatment of an iatrogenic thoracic aortic injury after spinal instrumentation: case report. J Vasc Surg. 2004;39(4):893-896. doi:10.1016/j.jvs.2003.10.056

15. Egea-Gámez RM, Wilson-MacDonald J, Murray D, González-Díaz R. Definition of safe zone in vertebral body in relation to anterior instrumentation. Spine Deformity. $2020 \mathrm{Mar}$ 13, p. 1-10. doi:10.1016/j.wneu.2020.03.054

All authors contributed substantially to the design of the work; data acquisition, analysis, and interpretation; drafting, revising, and approving the manuscript.

Disclosures and COI: The authors have no financial relationship(s) with a commercial interest producing health care goods or services. The present study has not been funded or received any grant and 
has not been published. This study was carried out in accordance with the World Medical Association Declaration of Helsinki (JBJS 79A:1089-98,1997). Patients' confidentiality was protected according to the US Health Insurance Portability and Accountability Act (HIPAA).

Corresponding Author: Rosa M. EgeaGámez, PhD, Hospital Universitario Niño Jesús, Orthopaedic Surgery, Spinal Unit, C/ Menéndez
Pelayo 65, 28009 JCR Madrid, Spain. Phone: +34 9150359 00; Fax: 915734012; Email: rosamaria. egea@salud.madrid.org

Published 30 June 2020

This manuscript is generously published free of charge by ISASS, the International Society for the Advancement of Spine Surgery. Copyright (C) 2020 ISASS. To see more or order reprints or permissions, see http://ijssurgery.com. 